\title{
La filiation et l'aide médicale à la procréation
}

\author{
Anne CADORET
}

CNRS-GRASS, Paris

\begin{abstract}
RESUME
La filiation suite à une insémination avec donneur inconnu dit les personnages retenus comme père et mère de l'enfant. Alors qu'il aurait été possible de construire cette filiation sur le modèle de l'adoption, le modèle choisi a été le don d'organes et de sang, qui efface complètement les donneurs afin que les receveurs puissent s'approprier totalement la substance donnée. Pourtant, avec le don de gamètes, s'agit-il de la même chose ? Le don de gamètes touche à la procréation d'un enfant tout en concernant la sexualité des parents. Pouvons-nous envisager que la sexualité des parents ne soit plus une sexualité procréative et que des figures de géniteur ou de génitrice puissent être reconnues à côté des figures de père et mère
\end{abstract}

Mots clés : filiation, adoption, dons, géniteurs, parents

\section{INTRODUCTION}

L'aide à la procréation, est définie comme une pratique clinique et biologique permettant une procréation en dehors du processus naturel, c'est-à-dire une procréation sans sexualité, tout en recherchant à établir une filiation aussi vraie que possible, aussi proche que possible d'une procréation " naturelle ». Que nous apprend, alors, l'insémination artificielle avec donneur (IAD) inconnu, sur notre conception de la parenté et sur ce que nous classons comme naturel dans la construction de la parenté ?

L'IAD va permettre une procréation aussi proche que possible du processus naturel de fabrication d'un enfant en s'appuyant sur les éléments de base de la procréation, soit, d'une part les substances (sperme et ovule) et d'autre part un processus corporel : la grossesse et l'accouchement. A partir de cette base technique de la procréation, chaque société va dire ce qu'elle retient comme incontournable pour fabriquer de l'humain entre les gamètes des parents et l'accouchement de la mère. Certaines vont demander que soit retenue au moins la substance d'un des parents, en l'occurrence le sperme ou l'ovocyte, mais interdire le prêt d'utérus (la mère porteuse), comme la France, alors que d'autres vont l'autoriser comme certains Etats des Etats-Unis ; ou encore, troisième possibilité, certaines sociétés vont permettre de faire appel à des gamètes autres que ceux du père et de la mère mais interdire la mère porteuse, comme la Catalogne : dans ce dernier cas la marque corporelle de la parenté repose uniquement sur la grossesse et l'accouchement.

En choisissant l'une ou l'autre formule, chaque société va déterminer qui, des donneurs et receveurs de corps, vont devenir les parents de l'enfant ou bien être rejetés de la parenté, transcrivant ainsi une donnée naturelle de la procréation en une donnée sociale : un père et/ou une mère.

C'est cette mise en place de la bonne parenté que je voudrais analyser.

\section{LE MODĖLE DE LA FILIATION ADOPTIVE}

Notre société occidentale nous a habitués à avoir deux parents et deux seuls parents, un père et une mère, miroir du masculin et féminin nécessaire à la production de l'espèce mammifère. Sur ces deux seuls parents reposent toutes les fonctions parentales de procréation, nourriture, éducation, transmission d'identité. La société occidentale nous a appris à voir dans les parents les géniteurs de l'enfant, ainsi que dans les géniteurs d'un enfant ses parents

Correspondance :

Anne CADORET - Cnrs-Grass, 59 rue Pouchet, 75017

Paris - Tel 01.40.25.13.16 - Fax 01.40.25.12.12 -

Email cadoret@iresco.fr 
comme si sexualité, procréation, alliance ou concubinage ne faisait qu'un pour donner la filiation. La filiation à partir d'une IAD demande alors de déconstruire une autre filiation possible : celle des personnages donneurs de substances corporelles (les gamètes, l'utérus). La solution retenue en France est d'effacer le donneur de substance et de refuser une mère porteuse, tout en instituant la femme accouchante - personnage toujours visible - comme seule mère possible ainsi que son conjoint ou concubin comme seul père.

Cependant, avant que I'IAD ne se mette en place, existait déjà une situation familiale dans laquelle les parents de l'enfant n'étaient pas ses géniteurs : celle de la filiation adoptive. La filiation suite à une IAD aurait donc pu être construite sur ce modèle déjà en cours. "Une bonne logique juridique aurait consisté à rattacher la filiation avec donneurs à la filiation adoptive, verrouillée par un jugement et assurant au parent stérile une parentalité définitive et non contestable. Mais, sous la pression du corps médical, une autre option législative a été prise : la filiation avec donneurs a été rattachée de manière fictive à la filiation charnelle (sans jugement par conséquent).» (Delaisi de Parseval : 115 [3]). En fait, ce mode d'établissement de la filiation s'est déjà trouvé utilisé pour régler un litige de filiation due à une erreur de transfert de gamètes ou d'embryon. Tyler [7] nous relate l'affaire telle qu'elle est dans les journaux britanniques pendant l'été 20021 .

Deux couples, un couple A, dont l'homme et la femme sont tous les deux de race blanche, et un couple $B$, dont l'homme et la femme sont tous les deux de race noire, demandent une fécondation in vitro avec transfert d'embryon, l'insémination se faisant pour chacun des couples avec leurs propres gamètes. Le couple A est tout heureux d'apprendre qu'il attend des jumeaux. Or la femme accouche de jumeaux de race noire. Soit, l'œuf fécondé du couple B fut, par erreur, transféré dans l'utérus d'une femme blanche, ou (scénario plus probable), le sperme de l'homme noir a été utilisé pour féconder l'œuf de la femme blanche... Quoiqu'il en soit, cette femme, Madame $A$, accouche de jumeaux noirs.

Normalement, rien n'aurait dû arriver, et la naissance aurait pu paraitre nature/le. Le couple blanc aurait dû avoir un enfant blanc (ils sont tous les deux les géniteurs) ; et si l'erreur avait été avec des gamètes d'un autre couple, mais de la même race, rien ne ce serait vu...

Toutefois, la naissance de ces jumeaux rend visible l'artificialité de leur conception, et il est difficile de ne pas en tenir compte pour établir la filiation et décider quels personnages de cette histoire seront appelés à devenir parents. Nous avons affaire à quatre parents potentiels : Madame $A$ qui porte ces jumeaux et accouche, Monsieur A qui a voulu un enfant de Madame A et a d'ailleurs donné son sperme pour cela. Monsieur $B$ qui a donné du sperme à une femme qu'il pense être Madame $B$; et enfin Madame $B$ qui veut aussi un enfant grâce au sperme de son époux, Monsieur B. Il est intéressant de s'arrêter sur les discussions - ou non discussions - suscitées par l'établissement de la filiation de ces enfants.
La détermination de la « bonne » mère ne pose pas problème : c'est Madame $A$, non tant parce qu'elle est la mère génétique des enfants que parce qu'elle les a portés pendant neuf mois.

Le père devrait alors, selon le Human Fertilisation and Embryology Act de 1990 être son mari, Monsieur A. Toutefois, s'il est prouvé que le père noir (Monsieur $B$ ) est géniteur2, il aurait des droits à la paternité, puisqu'il a donné son sperme pour devenir père de l'enfant né de ce don et non rester donneur.

Le verdict tombe en février 2003: Les test ADN établissent que $\mathrm{Mme} A$ est bien la mère biologique et $\mathrm{Mr} B$ le père biologique : le père noir qui est désigné comme le père légal des jumeaux, "aurait le droit d'aider à l'éducation des jumeaux". Cependant, la juge estime que les parents biologiques ( $\mathrm{Mr} B$ et Mme A) n'étant pas mariés l'un à l'autre et ne pouvant le faire, les enfants ne pourront avoir de relation avec leur père biologique (dixit Independent). II fut aussi décidé que le mari de Mme A devait adopter les enfants afin de reprendre le statut de père légal détenu par $\mathrm{Mr} B$. La relation matrimoniale de $\mathrm{Mr} A$ avec la mère génétique lui donne des droits sur le travail reproductif de cette dernière et défait toute revendication de parenté du père biologique.

Tyler remarque fort justement que si la paternité de Monsieur A est fondée sur son mariage avec Madame A, la réciproque n'est pas vraie pour Madame $B$ : alors qu'elle est l'épouse du père génétique - et légal à un moment de l'histoire - , elle n'a aucune légitimité juridique pour revendiquer un lien de parenté.

Ne retrouvons-nous pas, dans ce jugement, notre nœud gordien de la filiation découlant de l'alliance des père et mère, et encadrant la sexualité de la femme et la procréation, l'enfant que porte la femme devant être celui de son mari ?

\section{RÉFLEXIONS AUTOUR DES DONS DE SUBTANCE}

Toutefois, existe une différence entre l'adoption et l'IAD : dans l'adoption, le don ne porte pas sur une substance, mais sur un être humain déjà fabriqué, sur une personne déjà là, l'enfant. Cette différence de l'objet du don suffit-elle à justifier la différence de montage de la filiation ? Plutôt que d'une problèmatique de filiation, le don de gamètes pourrait-il relever d'une problèmatique de dons de substance, tels le don de sang et les dons d'organes ? Quelles similitudes et différences pourrions-nous établir entre tous ces dons ? Ou encore, comment s'y construit le lien entre donneur et receveur?

Notons d'abord une similitude générale « d'anonymiser » le donneur afin de mieux s'approprier, faire sienne la substance donnée [1]. En effet, si le don est le propre de relations personnelles tant à l'intérieur de communauté que de la famille, relations qui, de plus, relèvent de la moralité et du sentiment, l'anonymat, au contraire caractérise les grandes agglomérations urbaines, sans impliquer aucun type de relations personnelles ; il relève plutôt du domaine du mar- 
ché, du contrat, de l'intérêt et du calcul. Néanmoins, joindre ces deux termes opposés l'un à l'autre en parlant de don anonyme permet d'introduire des valeurs telles que l'altruisme sans pour autant entraîner des relations personnelles entre donneurs et receveurs, sauf exception 3 .

Toutefois, le sang reçu est difficilement identifiable pour le receveur puisque ce dernier reçoit du sang de plusieurs donneurs, sangs qui ne font plus qu'une même substance dans son corps. La question de " venir de ", de vivre grâce à un individu précis (même s'il est anonymisé) ne se pose pas vraiment. Alors que le don d'organe est parfaitement personnalisé, d'autant plus que l'organe reste un objet rare et non renouvelable. Il crée une lourde dette vis-à-vis du donneur mais aussi une difficulté de définition de soi puisque le " moi " est aussi un autre, qu'un intrus occupe son propre corps, que l'altérité est au plus profond de l'identité [6]. Un des moyens d'élaboration de cette nouvelle identité, tel que l'observe Fellous [4] dans son étude sur les greffés, est l'adoption : " L'organe, comme le serait un enfant dont les géniteurs biologiques ne sont pas les parents qui partagent son quotidien, est « à eux " et " pas à eux ». " (p.50).

Cependant, qui reçoit, lors des dons de gamètes ? Où est l'étrangeté à l'intérieur de soi ? Elle porte sur le parent et non sur l'enfant qui pourtant est le but du don. II me semble que c'est sur le déplacement de cette étrangeté qu'il faut réfléchir ; c'est cette étrangeté-là qu'interroge l'enfant quand on lui fait croire que ses origines viennent de ses parents, du corps de ses deux parents, comme si la relation sexuelle des parents, dans sa plus pure matérialité, devait être à l'origine du corps de leur enfant.

\section{PARENTÉ ET SEXUALITÉ}

Si la filiation de parents ayant recours à une insémination avec donneur ne s'est pas construite sur le modèle de la filiation adoptive, c'est qu'étant au plus près des corps, elle touche à la sexualité ainsi qu'à un ordre familial dans lequel sexualité, procréation et alliance se combinaient en un seul mouvement pour donner la filiation, ordre dans lequel père et mère se confondaient avec géniteur et génitrice.

Pourtant rien n'oblige à ce recouvrement entre sexualité et parenté. D'ailleurs, un certain nombre de sociétés nous montrent un montage de la filiation complètement différent :

Ainsi les $\mathrm{Na}$ de Chine [2], société complètement matrilinéaire, ont une cellule familiale formée de sœurs et frères, entre lesquels se dresse un interdit d'inceste total. Les femmes ont des amants "furtifs" de qui elles peuvent avoir des enfants, ces amants n'ayant aucune importance quant à la paternité de l'enfant. La filiation y est organisée sans recoupement avec la sexualité.

Ou encore, les Samo de Burkina Faso [5]; il s'agit d'une société patrilinéaire dans laquelle une femme ne va rejoindre son mari qu'après avoir eu une relation sexuelle avec un amant de son choix et avoir enfanté un enfant. Cependant le père de cet enfant sera non pas l'amant de la mère, mais son mari. De même, ce dernier restera le père de tous les enfants que cette femme pourra avoir d'unions secondaires. La raison de la filiation ne repose pas sur la sexualité.

Enfin, dernier exemple, la société Nuer, qui est aussi patrilinéaire. Une femme stérile peut épouser une autre femme pour laquelle son lignage a payé la dot en bétail, comme l'aurait fait un homme pour acquérir une épouse. Cette femme devient "le mari " de l'épouse et le "père " des enfants que cette dernière aura des œuvres d'un amant qui sera dédommagé de son travail de géniteur [5]. La filiation s'établit en dehors de toute sexualité procréative.

Dans ces exemples, on voit que sexualité, procréation et unité familiale ne se recoupent pas pour donner la filiation, chacun d'eux en proposant d'autres modes d'établissement.

\section{PROPOSITIONS}

D'ailleurs, dans notre propre société, nous assistons de plus en plus à une autonomisation de la procréation par rapport au mariage (ou au concubinage) et à la filiation avec l'augmentation du nombre de familles adoptives, recomposées, ayant recours à l'aide médicale à la procréation. Pouvons-nous encore croire que la procréation de l'enfant est la grande raison de sa filiation en perdurant dans une conception de deux seuls parents possibles, un père et une mère identiques au géniteur et à la génitrice, quels que soient les liens d'alliance entre eux deux ? Ou pouvonsnous concevoir que nous avons quelquefois quatre parents : un géniteur et un père, une génitrice et une mère ? Ou encore, qu'à côté de nos parents auxquels sont dévolus le rôle de parents et les fonctions parentales (la parentalité) existent d'autres figures nécessaires à notre venue au monde, les figures génitrices ? Qui font partie de la vérité biographique de l'enfant - car son corps vient en partie d'elles. Comment désigner toutes ces figures de parents et de géniteurs pour les distinguer les unes des autres ? II ne s'agit pas de nier le biologique ; le gène contient une vérité pour l'enfant qui le porte. II s'agit, seulement, de donner une place à ce biologique qui ne soit que la partie qui lui revient et ne peut recouvrir l'ensemble. Nos parents ne sont pas des clones de nos gènes. Une construction humaine n'est pas une vérité-objet, même si la connaissance de cette vérité-objet participe à cette construction de l'homme et de son identité familiale.

\section{RÉFÉRENCES}

1. BESTARD CAMPS J. : Tras la biología : La moralidad del parentesco y las nuevas tecnologías de reproducción. Barcelona, Estudis d'Antropologia Social i Cultural, 2005.

2. CAI H. : Une société sans père ni mari. Les Na de Chine. Paris, PUF, 1977.

3. DELAISI DE PARSEVAL G. : La pluriparentalité occultée : psychodynamique de la parentalité dans les cas d'aide médicale à la procréation avec dons de gamète. In : Le Gall D., Bettahar Y. eds. La Pluriparentalité. Paris, PUF, 2001 : 113124. 
4. FELLOUS M. : Soi-même et un autre : l'identité paradoxale du greffé, Cités, 2005, $21: 47-56$.

5. HERITIER F. : La cuisse de Jupiter. Réflexions sur les nouveaux modes de procréation. L'Homme, 1985, $94: 5-22$.

6. MARZANO M. : Lorsqu'un Intrus occupe le corps. Notes autour du livre de Jean-Luc Nancy. Cités, 2005, $21: 57-60$.

7. TYLER K. : Reflexions upon a racialised reprogenetic media event : the birth of " black " twins to a white IVF mother. Working papers, workshop 5, PUG- University of Manchester, 2003.

Communication au XIII $I^{\circ}$ Séminaire de la Fédération Française des CECOS, Tours, 7-9 Avril 2005.

Manuscrit reçu : mars 2005 ; accepté mai 2005.

Notes.

1 Dans le cadre d'un programme européen de recherche, intitulé "Public Understanding of Genetic » et piloté par IUniversité de Manchester.

2 Cette histoire nous montre bien la différence entre le statut de géniteur et le statut de donneur : l'un veut être père, l'autre non....

3 Des parents peuvent être appelés à donner un de leurs organes pour des raisons de compatibilité. L'article 671-3 de la loi de 1994 définissait cette proche parenté de la manière suivante: "Le receveur doit avoir la qualité de père ou de mère, de fils ou de fille, de frère ou de sœur du donneur". En cas d'urgence, le conjoint pouvait également se voir reconnaitre la qualité de donneur. Puis, à la suite de multiples débats, le cercle des donneurs potentiels fut élargi dans la loi votée en 2004.

\section{ABSTRACT}

Kinship and artificial insemination by unknown donor

\section{Anne CADORET}

Kinship following artificial insemination by unknown donor defines the child's mother and father as unknown. Although it could have been possible to construct this kinship on the model of adoption, the model adopted was that of blood and organ donation, which completely erases the donor's identity so that the recipient can take complete possession of the donated substance. But is gamete donation the same thing? Gamete donation concerns procreation and the parents' sexuality. Would it be possible to no longer consider the parents' sexuality to be exclusively reproductive sexuality allowing recognition of the progenitors alongside the mother and father?

Key-Words: kinship, adoption, donation, progenitors, parents 